University of Nebraska - Lincoln

DigitalCommons@University of Nebraska - Lincoln

Roger Kirby Publications

Research Papers in Physics and Astronomy

January 1972

Raman-Active Resonance Modes, Overtones, and Anharmonicity in $\mathrm{NaCl}: \mathrm{Cu}^{+}$

\author{
B.N. Ganguly \\ University of Nebraska - Lincoln \\ Roger D. Kirby \\ University of Nebraska-Lincoln, rkirby1@unl.edu
}

M.V. Klein

University of Nebraska - Lincoln

G.P. Montgomery Jr.

University of Nebraska - Lincoln

Follow this and additional works at: https://digitalcommons.unl.edu/physics_kirby

Part of the Physics Commons

Ganguly, B.N.; Kirby, Roger D.; Klein, M.V.; and Montgomery, G.P. Jr., "Raman-Active Resonance Modes, Overtones, and Anharmonicity in $\mathrm{NaCl}: \mathrm{Cu}^{+"}$ (1972). Roger Kirby Publications. 40.

https://digitalcommons.unl.edu/physics_kirby/40

This Article is brought to you for free and open access by the Research Papers in Physics and Astronomy at DigitalCommons@University of Nebraska - Lincoln. It has been accepted for inclusion in Roger Kirby Publications by an authorized administrator of DigitalCommons@University of Nebraska - Lincoln. 
(1965).

${ }^{13}$ C. M. Hurd and J. E. A. Alderson, Solid State Commun. 9, 309 (1971).

${ }^{14}$ Preliminary results in P. Monod and A. Friederich, in Proceedings of the Twelfth International Conference on Low Temperature Physics, Kyoto, 1970, edited by E. Kanda (Keigaku Publishing Co., Tokyo, 1971), p. 755.

${ }^{15} \mathrm{~A}$. Friederich and P. Monod, private communication. ${ }^{16}$ J. P. Burger, Ann. Phys. (Paris) $\underline{9}, 345$ (1964).

\title{
Raman-Active Resonance Modes, Overtones, and Anharmonicity in $\mathrm{NaCl}: \mathrm{Cu}^{+} \dagger$
}

\author{
B. N. Ganguly, R. D. Kirby,* M. V. Klein, and G. P. Montgomery, Jr. + \\ Department of Physics and Materials Research Laboratory, University of Illinois, Urbana, Illinois 61801
}

(Received 27 December 1971)

\begin{abstract}
The existence of an impurity-activated $E_{\mathrm{g}}$ resonance mode in $\mathrm{NaCl}: \mathrm{Cu}^{+}$has been suggested by several previous experiments. Raman data presented here reveal this resonance directly and also reveal the three components of the first overtone of the 23.5$\mathrm{cm}^{-1}$ infrared resonance mode. The frequencies of the $E_{\mathrm{g}}$ resonance and the $E_{\mathrm{g}} \mathrm{com}$ ponent of the overtone are shifted as a result of a strong anharmonic coupling. Their line shapes and strengths are considerably altered by an interference between the Raman amplitudes. A reasonable fit to the data has been obtained using a simple theory.
\end{abstract}

Substitutional impurities often introduce resonance modes into the lattice vibration spectrum of a crystal. ${ }^{1}$ Most experimental studies have been on infrared-active resonance modes. Raman-active modes have been predicted, but only in $\mathrm{KI}: \mathrm{Ag}^{+}$have they been found at very low frequency. ${ }^{2}$

The present work involves $\mathrm{NaCl}: \mathrm{Cu}^{+}$, which has been known for some time to have an infraredactive $\left(T_{1 \mathrm{u}}\right)$ resonance mode at $23.5 \mathrm{~cm}^{-1} \cdot{ }^{3}$ Its far-infrared properties have been studied under applied electric field $\mathbf{s}^{4}$ and uniaxial stress, ${ }^{5}$ and the isotope splitting has been resolved. ${ }^{6}$ It has a pronounced temperature dependence. ${ }^{3,7,8}$ The measured shift in peak position, increase in linewidth, and decrease in absorption strength with temperature could be explained by assuming the existence of an $E_{\mathrm{g}}$ resonance mode at about 31 $\mathrm{cm}^{-1}$, and coupling it anharmonically to the 23.5$\mathrm{cm}^{-1}$ mode. ${ }^{8}$ Additional indirect evidence for even-parity resonances in $\mathrm{NaCl}: \mathrm{Cu}^{+}$comes from thermal-conductivity measurements. The observed conductivity depression cannot be explained by the presence of the $23.5-\mathrm{cm}^{-1}$ resonance alone. ${ }^{9}$ It has been suggested that the data could be explained if an $E_{\mathrm{g}}$ resonance were present at a somewhat higher frequency. ${ }^{10}$

We now present direct evidence for such an $E_{\mathrm{g}}$ resonance mode. It is not seen in its "bare" harmonic form; it is strongly affected by an anharmonic interaction with a nearby $E_{\mathrm{g}}$ component of the first overtone of the $T_{1 u}$ mode. Raman data for all three first overtone modes will be presented to support this picture.
Figure 1 shows part of the Raman spectrum of $\mathrm{NaCl}: \mathrm{Cu}^{+}$taken with the $4880-\AA$ argon-laser line and a scattering geometry that yields all three Raman-active symmetries for the $O_{h}$ point group of the substitutional $\mathrm{Cu}^{+}$impurity. There are peaks at 40 and $48 \mathrm{~cm}^{-1}$ that are sensitive to temperature in a way reminiscent of the infrared mode. ${ }^{3,8}$ The $40-\mathrm{cm}^{-1}$ peak was originally interpreted as an ordinary $E_{\mathrm{g}}$ resonance mode. ${ }^{11} \mathrm{We}$ have made calculations using lattice Green's functions derived from realistic $\mathrm{NaCl}$ shell models that give an $E_{\mathrm{g}}$ resonance at about the correct frequency and width using force-constant

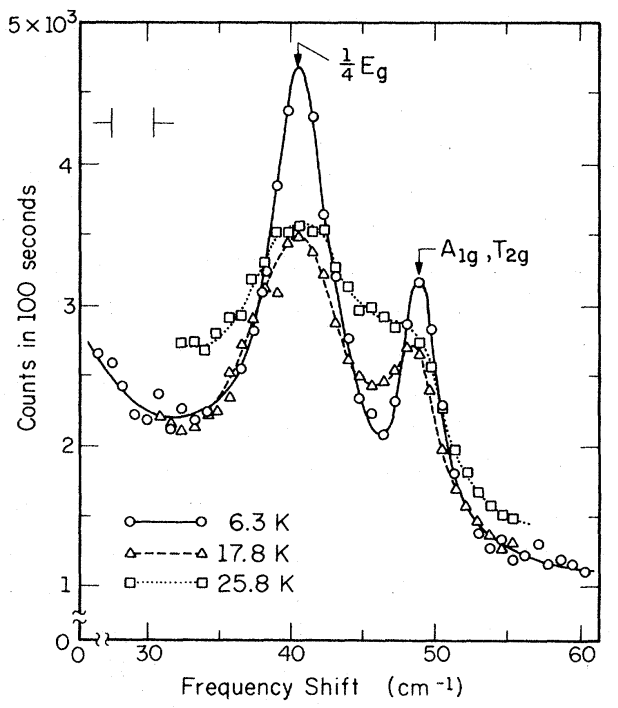

FIG. 1. Combined Raman spectrum of $\mathrm{NaCl}: \mathrm{Cu}^{+}$at moderate resolution showing strong temperature dependence. Copper concentration: $3 \times 10^{18} / \mathrm{cm}^{3}$. 
changes consistent with the existence of the infrared mode at $23.5 \mathrm{~cm}^{-1}$. The $A_{1 \mathrm{~g}}$ and $T_{2 \mathrm{~g}}$ peaks at $48 \mathrm{~cm}^{-1}$ cannot be so explained. If they were resonance modes, their widths should be even greater than that of the $40-\mathrm{cm}^{-1}$ mode. Furthermore, the existence of a $48-\mathrm{cm}^{-1} A_{1 \mathrm{~g}}$ resonance mode would require force constant changes sufficient to make the lattice unstable against $E_{\mathrm{g}}$ and $T_{1 \mathrm{u}}$ displacements.

We interpret the $48-\mathrm{cm}^{-1} T_{2 \mathrm{~g}}$ and $A_{1 \mathrm{~g}}$ peaks as two of the three components of the first overtone of the 23.5- $\mathrm{cm}^{-1} T_{1 \mathrm{u}}$ mode. The third, $E_{\mathrm{g}}$, component will be discussed below. Raman scattering from first overtones of an infrared-active ${ }^{\circ}-$ calized mode have been seen in alkaline-earth fluorides and alkali halides containing $\mathrm{H}^{-}$impurities. $^{12,13}$ Because of anharmonicity the three lines, $T_{2 \mathrm{~g}}, A_{1 \mathrm{~g}}$, and $E_{\mathrm{g}}$, occur at slightly different frequencies from each other and from twice the fundamental. In the present case our identification is based on the near factor of 2 in frequency and on similarities between the temperature dependence of the peak position and linewidth of the $48-\mathrm{cm}^{-1}$ line and the infrared line. This is shown for the $T_{2 g}$ component in Fig. 2. Within the experimental uncertainties, the widths and shift are consistent with the data shown in Fig. 3 of Ref. 7 for the $T_{1 u}$ mode if the assumption is made that the width of the overtone and its shift are twice those for the infrared mode.

Where then is the missing $E_{\mathrm{g}}$ component of the overtone? Figure 3 gives a detailed $E_{\mathrm{g}}$ Raman spectrum and shows that there are two peaks; the one at $40 \mathrm{~cm}^{-1}$ seen in Fig. 1 and a weak

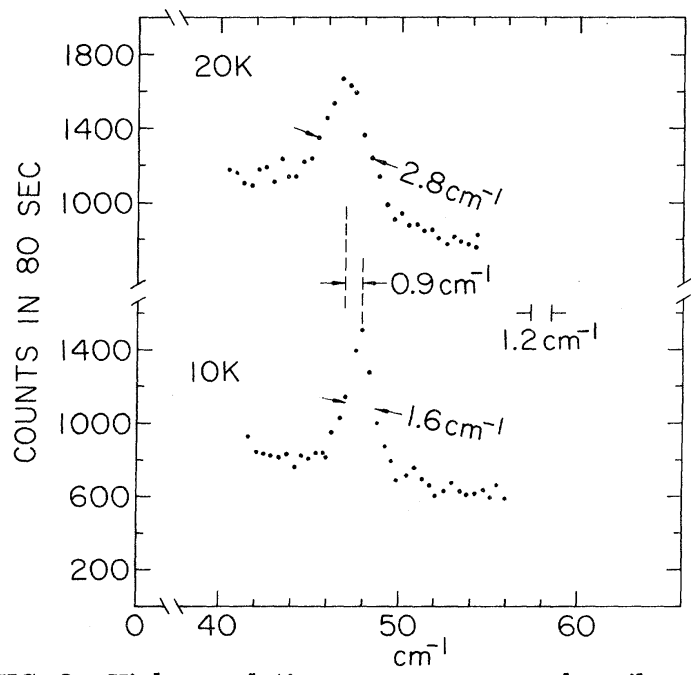

FIG. 2. High-resolution scan on a more heavily doped sample $\left(7.5 \times 10^{18} / \mathrm{cm}^{3}\right)$ of the $T_{2 \mathrm{~g}}$ component of the $48-\mathrm{cm}^{-1}$ peak from Fig. 1 . asymmetric second peak at $63 \mathrm{~cm}^{-1}$. We believe that these peaks result from anharmonic coupling and mixing of an $E_{\mathrm{g}}$ resonance mode and the $E_{\mathrm{g}}$ component of the first overtone of the $T_{1 \mathrm{u}}$ mode.

To make a preliminary analysis of this coupling, we let (1) and 12) denote excited states corresponding to the $E_{\mathrm{g}}$ resonance and $E_{\mathrm{g}}$ overtone, respectively, $\omega_{1}$ and $\omega_{2}$ the corresponding unperturbed frequencies, and $V$ the matrix element of the anharmonic perturbation coupling them. Then in a system of units where $\hbar=1$, the Hamiltonian $H$ has matrix elements $(1|H| 1)=\omega_{1}$, $(2|H| 2)=\omega_{2}$, and $(1|H| 2)=(2|H| 1)^{*}=V$. Its eigenvalues $\lambda_{1}$ and $\lambda_{2}$ obey the equations

$$
\begin{aligned}
& \lambda_{1}+\lambda_{2}=\omega_{1}+\omega_{2}, \\
& \lambda_{1} \lambda_{2}=\omega_{1} \omega_{2}-|V|^{2} .
\end{aligned}
$$

Inserting $\omega_{2}=48 \mathrm{~cm}^{-1}, \lambda_{1}=40 \mathrm{~cm}^{-1}, \lambda_{2}=63 \mathrm{~cm}^{-1}$, we find from Eqs. (1) and (2)

$$
\begin{aligned}
& \omega_{1}=55 \mathrm{~cm}^{-1}, \\
& |V|=11 \mathrm{~cm}^{-1} .
\end{aligned}
$$

Note that the $63-\mathrm{cm}^{-1}$ peak in Fig. 3 is asymmetric and that there is a distinct minimum at $54.6 \mathrm{~cm}^{-1}$ (arrow) that could well correspond to a zero in intensity. These features are strongly suggestive of an interference process. A discussion of this interference requires a calculation of the line shape for the coupled levels. ${ }^{14}$ Let the amplitude for a Raman transition to unperturbed state 11) be $P_{1}$ and to unperturbed state 12) be

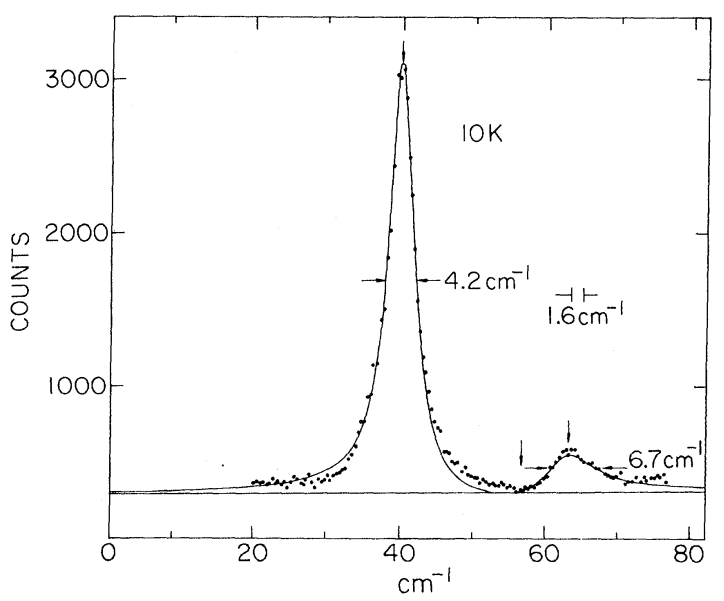

FIG. 3. High-resolution scan of the $E_{\mathrm{g}}$ Raman spectrum. The ordinate is the number of counts in $20 \mathrm{sec}$ below $48 \mathrm{~cm}^{-1}$ and one half the number of counts in 40 sec above $48 \mathrm{~cm}^{-1}$. The solid line is a theoretical curve described in the text. Copper concentration: $7.5 \times 10^{18} / \mathrm{cm}^{3}$. 
$P_{2}$. The $P$ 's will be proportional to appropriate polarizability derivatives. The low-temperature Stokes-Raman intensity due to the coupled modes at frequencies $\lambda_{1}, \lambda_{2}$ may be obtained by rewriting the energy $\delta$ function in Fermi's "golden rule" to yield

$$
W(\omega)=\operatorname{Im} \sum_{i, j=1,2} P_{i} *(i|G| j) P_{j},
$$

where the operator $G \equiv(H-\omega-i \epsilon)^{-1}, \epsilon \rightarrow 0^{+}$, can be shown to have matrix elements

$$
\begin{aligned}
& (1|G| 1)=\left(1\left|G_{0}\right| 1\right) D^{-1}, \\
& (2|G| 2)=\left(2\left|G_{0}\right| 2\right) D^{-1}, \\
& (2|G| 1)^{*}=(1|G| 2)=-\left(1\left|G_{0}\right| 1\right)\left(2\left|G_{0}\right| 2\right) V D^{-1},
\end{aligned}
$$

with

$$
D=1-\left(1\left|G_{0}\right| 1\right)\left(2\left|G_{0}\right| 2\right)|V|^{2} .
$$

We assume that the unperturbed $E_{\mathrm{g}}$ resonance level has a finite halfwidth at half-maximum denoted by $\gamma$ and that the unperturbed second harmonic is very narrow. This gives

$$
\begin{aligned}
& \left(1\left|G_{0}\right| 1\right)=\left(\omega_{1}-\omega-i \gamma\right)^{-1}, \\
& \left(2\left|G_{0}\right| 2\right)=\left(\omega_{2}-\omega-i \epsilon\right)^{-1},
\end{aligned}
$$

and

$$
W(\omega)=\frac{\left|P_{1}\right|^{2} \gamma\left(\omega-\omega_{0}\right)^{2}}{\left(\lambda_{1}-\omega\right)^{2}\left(\lambda_{2}-\omega\right)^{2}+\gamma^{2}\left(\omega_{2}-\omega\right)^{2}},
$$

where $\lambda_{1,2}$ obey Eqs. (1), (2) and where

$$
\omega_{0} \equiv \omega_{2}-P_{2} V / P_{1} \text {. }
$$

We have assumed that $P_{2} V / P_{1}$ is a real number.

The solid line in Fig. 3 is calculated from Eq. (9) with $\lambda_{1}=40 \mathrm{~cm}^{-1}, \lambda_{2}=62.5 \mathrm{~cm}^{-1}, \gamma=5.9 \mathrm{~cm}^{-1}$, and $\omega_{0}=54.8 \mathrm{~cm}^{-1}$. The fit is not perfect, but it represents a good compromise involving the relative peak heights, shapes, and the position of the minimum. Equations (1) and (2) then give

$$
\begin{aligned}
& \omega_{1}=54.5 \mathrm{~cm}^{-1}, \\
& V=10.8 \mathrm{~cm}^{-1}
\end{aligned}
$$

( $V$ assumed real and positive), ${ }^{15}$ and Eq. (10) then gives

$$
P_{2} / P_{1}=-0.63
$$

The wave functions for the perturbed modes are readily calculated and yield the result that the $40-$ $\mathrm{cm}^{-1}$ mode is a mixture of $65 \% E_{\mathrm{g}}$ overtone and $35 \% E_{\mathrm{g}}$ resonance, whereas for the $62.5-\mathrm{cm}^{-1}$ mode these figures are reversed. The strength ratio 65:35 is strongly modified in the Raman spectrum by the $\left(\omega-\omega_{0}\right)^{2}$ factor in Eq. (9). This factor describes the result of the interference be- tween the $P_{1}$ amplitude and the $P_{2}$ amplitude due to the anharmonic coupling provided by $V$.

If there were no anharmonic coupling, there would be an $E_{\mathrm{g}}$ resonance mode at $54.5 \mathrm{~cm}^{-1}$ with a full width at half-maximum of $10.8 \mathrm{~cm}^{-1}$ and a peak height $29 \%$ of the height of the $40-\mathrm{cm}^{-1}$ peak in Fig. 3. The $E_{\mathrm{g}}$ component of the overtone at $48 \mathrm{~cm}^{-1}$ would have a width comparable to that of the $T_{2 \mathrm{~g}}$ overtone shown in Fig. 2 and an integrated area equal to $\left(P_{2} / P_{1}\right)=40 \%$ of that of the unperturbed $E_{\mathrm{g}}$ peak. The total area under the two unperturbed peaks would equal the area of the perturbed spectrum in Fig. 3.

Additional evidence for strong anharmonic coupling in $\mathrm{NaCl}: \mathrm{Cu}^{+}$is provided by the existence of combination bands in the far infrared at 64 and $69 \mathrm{~cm}^{-1} .^{11}$ A paper containing detailed discussions of the infrared work and of additional $\mathrm{Ra}-$ man results will be forthcoming.

We thank R. F. Wood for discussions about lattice Green's functions.

†Work supported in part by the National Science Foundation under Contracts No. GP 6581, No. 11173, and No. 28319, and by the Advanced Research Projects Agency under Contract No. HC15-67-C-0221.

*Present address: Behlen Laboratory, Department of Physics, University of Nebraska, Lincoln, Neb. 68508.

$\$$ Present address: Code 6510, U. S. Naval Research Laboratory, Washington, D. C. 20390.

${ }^{1}$ M. V. Klein, in Physics of Color Centers, edited by W. B. Fowler (Academic, New York, 1968), Chap. 7.

${ }^{2}$ R. D. Kirby, Phys. Rev. B $\underline{4}, 3557$ (1971).

${ }^{3}$ R. Weber and P. Nette, Phys. Lett. 20, 493 (1966).

${ }^{4}$ B. P. Clayman, R. D. Kirby, and A. J. Sievers, Phys. Rev. B $\underline{3}, 1351$ (1971).

${ }^{5}$ G. Busse, W. Prettl, and L. Genzel, Phys. Lett. 27A, 438 (1968).

${ }^{6}$ R. D. Kirby, I. G. Nolt, R. W. Alexander, Jr., and A. J. Sievers, Phys. Rev. 168, 1057 (1968).

${ }^{7}$ R. Weber and F. Siebert, Z. Phys. 213, 273 (1968).

${ }^{8}$ R. W. Alexander, Jr., A. E. Hughes, and A. J. Sievers, Phys. Rev. B 1, 1563 (1970).

${ }^{9}$ R. F. Caldwell and M. V. Klein, Phys. Rev. $\underline{158}, 851$ (1967).

${ }^{10}$ F. Baumann and R. O. Pohl, Phys. Rev. 163,843 (1967).

${ }^{11}$ G. P. Montgomery, Jr., and R. D. Kirby, in Light Scattering in Solids, edited by M. Balkanski (Flammarion, Paris, 1971).

${ }^{12} \mathrm{~J}$. A. Harrington, R. T. Harley, and C. T. Walker, Solid State Commun. 8 , 407 (1970).

${ }^{13}$ G. P. Montgomery, Jr., W. R. Fenner, M. V. Klein, and T. Timusk, Phys. Rev. B (to be published).

${ }^{14}$ R. S. Katiyar, J. F. Ryan, and J. F. Scott, Phys. Rev. B 4 , 2635 (1971).

${ }^{15}$ This value implies that the third-order anharmonicity parameter $V_{E}$ introduced in Eq. (3) of Ref. 2 would have a value of about $7.4 \times 10^{11} \mathrm{erg} / \mathrm{cm}^{3}$. 